\title{
ÜBER DIE TEILUNG VON EBENEN KONVEXEN BEREICHEN DURCH SEHNEN
}

\author{
ARNE PLEIJEL
}

Es sei $c$ eine geschlossene konvexe Kurve und $g$ eine sie schneidende Gerade. Wir bezeichnen mit $F_{1}, F_{2}$ die Flächeninhalte der beiden von $c$ und $g$ begrenzten Gebiete und mit $L_{1}, L_{2}$ die Längen der entsprechenden Teilbögen von $c$. Ferner setzen wir $F_{1} / F_{2}=k, L_{1} / L_{2}=q$. Die vorliegende Arbeit beschäftigt sich mit der Frage, ob es möglich ist, bei vorgegebenem $k$ Schranken für $q$ anzugeben, die von der Gestalt von $c$ und der Lage von $g$ unabhängig sind. Es zeigt sich, dass dies in der Tat möglich ist, und zwar gilt

$$
1+2 k>q>k /(2+k) .
$$

Ist die eine dieser beiden Ungleichungen allgemein gültig, so folgt aus ihr die andere durch Vertauschung der Teilgebiete. Wir brauchen somit nur einen Beweis für die erste Ungleichung zu erbringen.

Diesem Beweise liegt folgender Gedanke zugrunde. Legt man durch die Schnittpunkte $B, C$ der Geraden $g$ mit der Kurve $c$ je eine Stützgerade, so liegt die Kurve ganz innerhalb des von ihnen gebildeten Winkels (oder Parallelstreifens). Man betrachtet nun eine zweite geschlossene Kurve $\gamma$, welche ebenfalls durch $B$ und $C$ geht und innerhalb desselben Winkels (bzw. Parallelstreifens) liegt. Das von $\gamma$ umschlossene Gebiet wird dann von der Geraden $B C=g$ ebenfalls in zwei Teile zerlegt. Es seien $f_{1}, f_{2}$ die Flächeninhalte dieser Teilgebiete und $l_{1}, l_{2}$ die Längen der entsprechenden Teilbögen von $\gamma$. Wenn es nun möglich ist, diese Kurve $\gamma$ so zu wählen, dass die Beziehungen $f_{1}=F_{1}, f_{2}=F_{2}, l_{1} \geqq L_{1}, l_{2} \leqq L_{2}$ gelten, und wenn sich zeigen lässt, dass $1+2 f_{1} / f_{2}>l_{1} / l_{2}$ ist, so muss wegen $f_{1} / f_{2}=F_{1} / F_{2}=k$ und $l_{1} / l_{2} \geqq L_{1} / L_{2}=q$ die Ungleichung $1+2 k>q$ a fortiori gelten. Diese Ungleichung ist in dem Sinne scharf, dass $1+2 k-q$ der Null beliebig nahe kommen kann. Andererseits lässt sie sich, wie abschliessend gezeigt wird, zu

$$
1+2 k-q>\alpha F^{2} L^{-4}
$$

Eingegangen am 13. November 1953. 


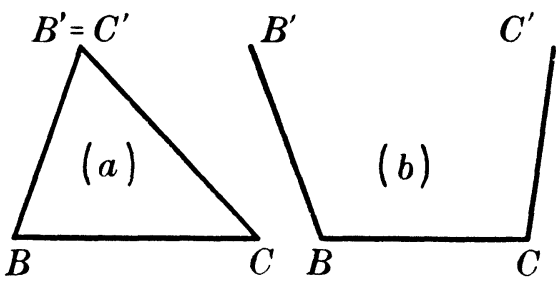

Fig. 1.

verschärfen, wo $F$ und $L$ den Inhalt bzw. Umfang des ganzen Bereichs bezeichnen und $\alpha$ eine positive Konstante ist.

Die Aufgabe, eine Kurve $\gamma$ mit den genannten Eigenschaften zu finden, führt auf das folgende Problem. Es sei $\Omega$ ein von einer Strecke $B C$ und zwei von $B$ und $C$ ausgehenden Strecken oder Halbstrahlen begrenztes Gebiet; es kommen zwei Gestalten für $\Omega$ in Frage, die wir mit $(a)$ bzw. (b) bezeichnen wollen (vgl. Fig. 1). Man betrachtet nun alle ganz in $\Omega$ liegenden konvexen Bereiche vorgegebenen Inhalts, deren Begrenzungen die Strecke $B C$ enthalten; gefragt wird, welcher von diesen Bereichen kleinsten bzw. grössten Umfang hat.

1. Wir beschäftigen uns zunächst mit dem zuletzt genannten Maximumproblem. Nach Favard [2] wird dieses für die Gebiete der Form (a) durch ein Dreieck $B C D$ gelöst, dessen dritter Eckpunkt $D$ auf $B B^{\prime}$ liegt,

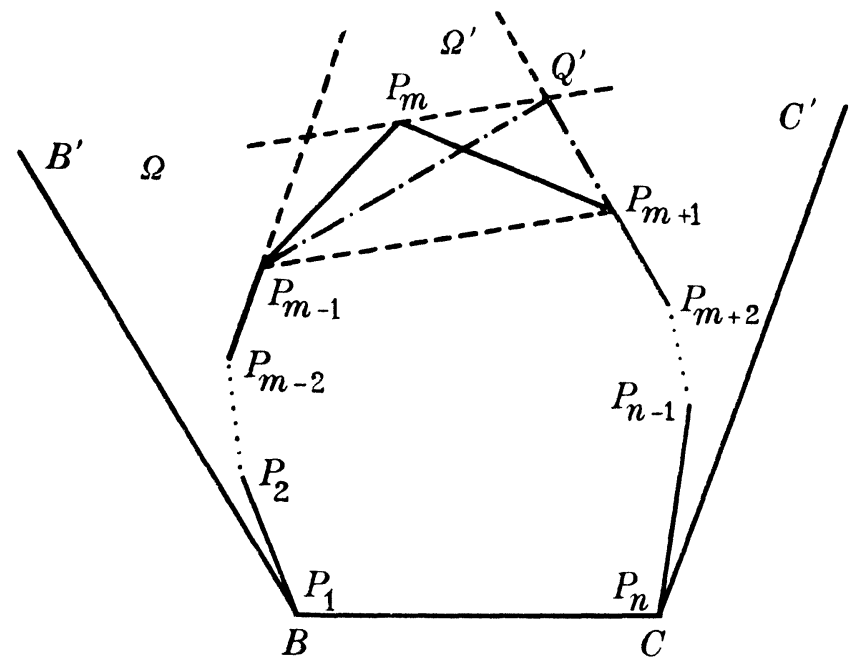

Fig. 2.

wenn die Bezeichnungen so gewählt sind, dass der Winkel $B^{\prime} B C$ nicht kleiner als $B C C^{\prime}$ ist. Die Schlussweise von Favard kann auch auf die Form $(b)$ angewendet werden. Man kann aber auch folgendermassen vorgehen. Da man eine konvexe Kurve beliebig genau durch konvexe Polygone approximieren kann, genügt es, im Beweis für die Extremaleigenschaft solche zu betrachten. Wir haben also ein Gebiet $\Omega$ und darin 
ein konvexes Polygon $P_{1} P_{2} \ldots P_{n}$, wobei etwa $P_{1}=B$ und $P_{n}=C$ ist. Wir wählen eine Ecke $P_{m}, 1<m \leqq n-1$, und betrachten das Dreieck $P_{m-1} P_{m} P_{m+1}$ und das Gebiet $\Omega^{\prime}$, das von der Strecke $P_{m-1} P_{m+1}$ und von den Verlängerungen der Seiten $P_{m-2} P_{m-1}$ und $P_{m+2} P_{m+1}$ begrenzt wird. Dieses Gebiet liegt wegen der Konvexität des Polygons sicher ganz in dem Gebiet $\Omega$. Unter den ganz in $\Omega^{\prime}$ gelegenen, zu $P_{m-1} P_{m} P_{m+1}$ inhaltsgleichen Dreiecken $P_{m-1} Q P_{m+1}$ sei $P_{m-1} Q^{\prime} P_{m+1}$ dasjenige mit dem grössten Umfang (vgl. Fig. 2). Die dritte Ecke dieses Dreiecks muss dann auf einem der beiden Halbstrahlen liegen, die $\Omega^{\prime}$ begrenzen und zwar auf demjenigen, der mit der Geraden $P_{m-1} P_{m+1}$ den grösseren Winkel bildet. Durch diese Konstruktion haben wir ein konvexes Polygon $P_{1} \ldots P_{n}$ mit gleichem Flächeninhalt aber grösserem Umfang erhalten, und dieses Polygon enthält eine Ecke weniger als das ursprüngliche. Dieses neue Polygon liegt ebenfalls ganz innerhalb des Gebietes $\Omega$. Durch Wiederholung dieser Konstruktion können wir nun die Anzahl der Ecken so lange verkleinern, bis nur drei übrig bleiben, von denen zwei sicher mit $B$ und $C$ zusammenfallen. Durch eine nochmalige Anwendung der Konstruktion bekommen wir das oben angegebene Dreieck.

Für eine beliebige, in $\Omega$ gelegene, durch $B$ und $C$ gehende konvexe Kurve können wir nun hieraus nur schliessen, dass dieses Dreieck mindestens den gleichen Umfang hat, jedoch nicht, dass es der einzige konvexe Bereich ist, für welchen diese obere Grenze erreicht wird. Um nun zu zeigen, dass dies auch für beliebige konvexe Kurven gilt, betrachten wir den Punkt (oder einen der Punkte) $E$ der gegebenen konvexen Kurve, der die grösste Entfernung von der Geraden $B C$ hat. Durch diesen Punkt ziehen wir eine Parallele zu $B C$; sie schneide $B B^{\prime}$ im Punkte $E_{1}$ und $C C^{\prime}$ im Punkte $E_{2}$. Wir verbinden $E$ mit den Punkten $B$ und $C$ und wenden das gefundene Resultat auf die beiden Teile der konvexen Kurve an, die innerhalb der Dreiecke $E B E_{1}$ bzw. $E C E_{2}$ liegen. Wir erhalten so ein konvexes Vieleck, das mindestens den gleichen Umfang und denselben Flächeninhalt wie die gegebene Kurve hat. Der Umfang dieses Vielecks ist aber sicher kleiner als der Umfang des Dreiecks $B D C$, es sei denn, dass die gegebene Kurve mit dem Dreieck $B D C$ zusammenfällt; denn nur in diesem Falle kann die Entfernung der Geraden $B C$ und $E E_{1}$ der Entfernung des Punktes $D$ von $B C$ gleich sein. Es gilt also:

Unter allen inhaltsgleichen konvexen Bereichen, deren Ränder die Strecke $B C$ enthalten und die ganz in einem Gebiet $\Omega$ enthalten sind, hat ein Dreieck $B D C$ den grössten Umfang. Die Ecke $D$ dieses Dreiecks liegt auf dem Rand von $\Omega$, und zwar auf $B B^{\prime}$, wenn Winkel $C B B^{\prime}$ grösser als Winkel $B C C^{\prime}$ ist. Das Dreieck ist somit eindeutig bestimmt, falls diese Winkel nicht einander gleich sind. 
2. Ich gehe jetzt zum Minimumproblem über und beweise folgenden Satz (der in der zitierten Arbeit von Favard ohne Beweis erwähnt ist):

Unter allen inhaltsgleichen konvexen Bereichen, deren Ränder die Strecke $B C$ enthalten und die ganz in einem Gebiet $\Omega$ (vgl. Fig. 1) liegen, gibt es einen mit minimalem Umfang. Dieser Minimalbereich ist eindeutig bestimmt, abgesehen von dem Fall, wo in $\Omega$ die Winkel bei $B$ und $C$ einander gleich sind, in welchem es zwei symmetrische Minimalbereiche gibt. Werden die Bezeichnungen so gewählt, dass der Winkel $C$ grösser oder gleich dem Winkel B ist, so besteht der Rand des Minimalbereichs aus der Strecke BC und ausserdem (je nach der Grösse des Flächeninhalts):

$1^{\circ}$ aus einem Kreisbogen, der $B$ mit $C$ verbindet, oder

$2^{\circ}$ aus einem von $C$ ausgehenden Kreisbogen, der $B B^{\prime}$ in einem Punkt $E$ berührt, und aus der Strecke $E B$, oder

$3^{\circ}$ aus einem Kreisbogen, der $B B^{\prime}$ in einem Punkt $F$ und $C C^{\prime}$ in einem Punkt $G$ berïhrt, und aus den Strecken $F B$ und $G C$.

Um dies einzusehen, bemerken wir zunächst, dass von zwei konvexen Bereichen, von denen der eine ganz innerhalb des anderen liegt (ev. mit Ausnahme der Teile der Begrenzung, die auch zur Begrenzung von $\Omega$ gehören) der innere sowohl kleineren Umfang als auch kleineren Flächeninhalt hat. Ferner erinnern wir an die folgende wohlbekannte Tatsache. Unter allen inhaltsgleichen konvexen Bereichen, deren Ränder eine feste Strecke enthalten, hat das Kreissegment mit der Strecke als begrenzender Sehne den kleinsten Umfang. Wir können dann so schliessen: Da die Strecke $B C$ im Umfang enthalten ist, muss die Menge der konvexen Kurven, die betrachtet wird, gleichmässig beschränkt sein. Nach dem Auswahlsatz von Blaschke [1, S. 62] existiert dann eine konvexe Kurve, für die das Minimum des Umfangs erreicht wird. Diese Minimumkurve muss dann mit Ausnahme der Teile, die der Begrenzung des Gebiets $\Omega$ angehören, aus einem Kreisbogen bestehen. Denn gäbe es einen Teilbogen $H K$, der kein Kreisbogen wäre, so könnte man über der Sehne $H K$ einen Kreisbogen konstruieren, der zusammen mit dem übrigen Teil der früheren Kurve der Minimumkurve inhaltsgleich wäre. Die neue Kurve hätte dann kleineren Umfang als die ursprüngliche. Sie ist zwar nicht notwendig konvex, aber ihre konvexe Hülle hätte grösseren Inhalt und noch kleineren Umfang und liegt, wenn nur der Bogen $H K$ hinreichend klein genommen wird, innerhalb des Gebiets $\Omega$. Dies widerspricht der Annahme, dass die ursprüngliche Kurve eine Minimalkurve war. Man sieht also, dass die letztere aus Teilen des Randes von $\Omega$ und einem Kreisbogen bestehen muss. Dass dieser Kreisbogen in dem auf $B B^{\prime}$ bzw. $C C^{\prime}$ gelegenen Endpunkt, falls dieser nicht mit $B$ bzw. $C$ zusammen- 
fällt, $B B^{\prime}$ bzw. $C C^{\prime}$ berühren muss, sieht man folgendermassen ein. Sei etwa der auf $B B^{\prime}$ gelegene Endpunkt $L$ von $B$ verschieden. Wir nehmen an, dass dort keine Berührung stattfindet. Es seien $M$ ein Punkt des Kreisbogens und $L^{\prime}$ ein Punkt auf $B B^{\prime}$ zwischen $B$ und $L$. Wir ersetzen nun das Kurvenstück $L^{\prime} L M$ durch denjenigen $L^{\prime}$ und $M$ verbindenden Kreisbogen, der mit der Strecke $L^{\prime} M$ denselben Flächeninhalt wie das Kurvenstück $L^{\prime} L M$ einschliesst. Wählen wir $L^{\prime}$ hinreichend nahe bei $L$, so liegt aus Stetigkeitsgründen auch die neue Kurve in $\Omega$, und da diese denselben Inhalt aber kleineren Umfang als die ursprüngliche hat, sind wir nochmals zu einem Widerspruch gelangt. Da es nun zu jedem gegebenen Flächeninhalt eine und nur eine Figur der im Satz angegebenen Form gibt, ist hiermit die Behauptung vollständig bewiesen.

3. Mit Hilfe der obigen Sätze soll nun die erste, in der Einleitung genannte Aufgabe gelöst werden. Der zu beweisende Satz lautet:

Teilt eine Gerade einen konvexen Bereich in Teile mit den Flächeninhalten $F_{1}$ und $F_{2}$, und sind $L_{1}$ und $L_{2}$ die Längen der entsprechenden Bögen, in die die Randkurve geteilt wird, so gilt

$$
1+2 k-q>0,
$$

wo $F_{1} / F_{2}=k$ und $L_{1} / L_{2}=q$ gesetzt ist. Die Null der rechten Seite kann hierin durch keine positive Konstante ersetzt werden.

Seien also ein konvexer Bereich $\Gamma$ und eine ihn schneidende Gerade $g$ gegeben. Durch die Schnittpunkte $B$ und $C$ von $g$ mit der Randkurve von $\Gamma$ legen wir Stützgeraden $B^{\prime} B B^{\prime \prime}$ und $C^{\prime} C C^{\prime \prime}$, wo die Bezeichnungen so gewählt seien, dass $B^{\prime}$ und $C^{\prime}$ auf derselben Seite von $g$ liegen und dass Winkel $B^{\prime} B C$ grösser als oder gleich Winkel $C^{\prime} C B$ ist. Auf diese Weise erhalten wir zwei Gebiete $B^{\prime} B C C^{\prime}$ und $B^{\prime \prime} B C C^{\prime \prime}$ von der in der Einleitung mit $\Omega$ bezeichneten Art (vgl. Fig. 1). Es sei $F_{1}$ bzw. $F_{2}$ der .Inhalt des im Bereich $B^{\prime} B C C^{\prime}$ bzw. $B^{\prime \prime} B C C^{\prime \prime}$ gelegenen Teils von $\Gamma$, und $L_{1}$ und $L_{2}$ seien die Längen der entsprechenden Teilbögen der Randkurve. Wir ersetzen nun den in $B^{\prime} B C C^{\prime}$ gelegenen Teil von $\Gamma$ durch dasjenige Dreieck $B C P$ mit demselben Inhalt $F_{1}$, dessen Ecke $P$ auf $B B^{\prime}$ liegt. Nach $A b$ schnitt 1 gilt dann $B P+P C \geqq L_{1}$. Wir verlängern nun $P C$ über $C$ hinaus und bezeichnen die entstehende Halbgerade mit $C Q$. Dadurch entsteht ein Gebiet $B^{\prime \prime} B C Q$, das den in $B^{\prime \prime} B C C^{\prime \prime}$ gelegenen Teil von $\Gamma$ enthält. Diesen Teil ersetzen wir nach Abschnitt 2 durch die in $B^{\prime \prime} B C Q$ enthaltene Figur mit demselben Inhalt $\boldsymbol{F}_{2}$ und minimalem Umfang. Damit ist $\Gamma$ durch eine Figur ersetzt, die eine der drei in Figur 3 angegebenen Formen hat und für die $F_{1}$ und $F_{2}$ dieselben Werte wie für $\Gamma$ 
haben, während $L_{1}$ nicht verkleinert und $L_{2}$ nicht vergrössert ist. Wenn wir jetzt zeigen können, dass die behauptete Ungleichung für den neuen Bereich gilt, so muss sie a fortiori für $\Gamma$ gelten.

Es genügt aber den Fall der Figur $3 \mathrm{~b}$ zu betrachten; denn die beiden anderen können auf diesen zurückgeführt werden. Dies geschieht auf folgende Weise. Im Fall der Figur $3 \mathrm{a}$, die aus einem Kreissegment

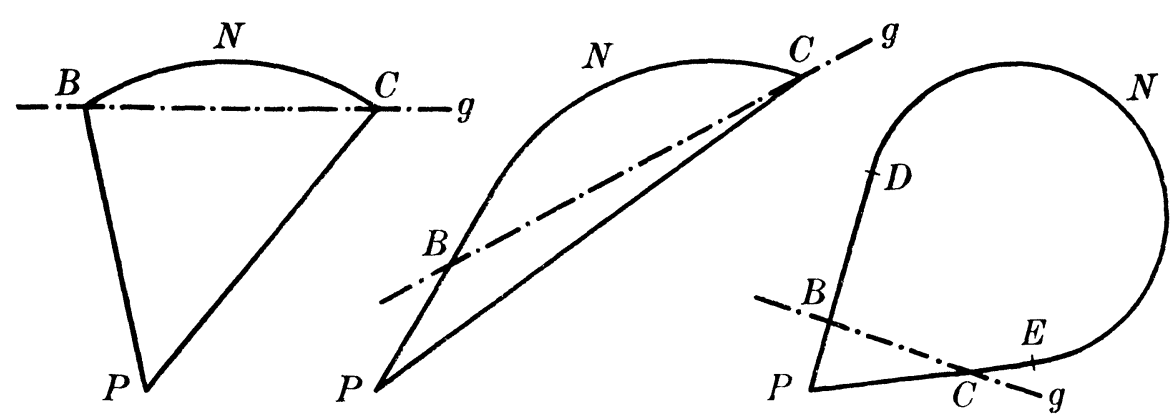

Fig. 3 a.

Fig 3 b.

$B N C$ und einem Dreieck $B C P$ besteht, ersetzen wir das Dreieck durch dasjenige inhaltsgleiche Dreieck $B C P^{\prime}$, dessen Ecke $P^{\prime}$ auf der Tangente des Kreisbogens in $B$ (auf derselben Seite von $g$ wie $P$ ) liegt. Hierdurch wird $L_{1}$ weiter vergrössert, während $F_{1}, F_{2}$ und $L_{2}$ ungeändert bleiben. Die entstehende Figur ist offensichtlich ein Spezialfall von Figur $3 \mathrm{~b}$. Der Bereich der Figur 3c wird von einem Kreisbogen $D N E$ und den Tangenten DBP und ECP in seinen Endpunkten berandet. Ohne Beschränkung der Allgemeinheit können wir annehmen, dass $B P \leqq C P$ ist. Wir ändern nun unter Festhaltung des Flächeninhalts des durch $g$ abgeschnittenen Dreiecks $B C P$ die Lage der Geraden $g=B C$ derart, dass sie schliesslich durch $E$ geht und $D P$ etwa in $B_{1}$ schneidet. Dann ist die Summe $B_{1} P+P E$ der nun von den Tangenten abgeschnittenen Strecken grösser als die ursprüngliche $B P+P C$, da die Summe zweier Variablen, deren Produkt konstant ist, ihr Minimum erreicht, wenn die Variablen einander gleich sind, und monoton wächst, wenn die Variablen sich von diesem gemeinsamen Wert entfernen. Es ist also $L_{1}$ wieder vergrössert und $L_{2}$ entsprechend verkleinert worden, während $F_{1}$ und $F_{2}$ ungeändert geblieben sind. Es genügt also auch hier, die behauptete Ungleichung für die neue Figur, die die Gestalt der Figur $3 \mathrm{~b}$ hat, zu beweisen.

Zusammenfassend können wir also sagen: $\mathrm{Zu}$ jedem konvexen Bereich, dessen Inhalt bzw. Umfang durch eine Gerade $g$ in Teile $F_{1}$ und $F_{2}$ bzw. $L_{1}$ und $L_{2}$ geteilt wird, lässt sich ein inhaltsgleicher Bereich von der in Figur $3 \mathrm{~b}$ dargestellten Form bestimmen, der durch $g$ ebenfalls in Teile 
mit den Inhalten $F_{1}$ und $F_{2}$ geteilt wird und dessen entsprechende Umfangsteile $l_{1}$ und $l_{2}$ den Ungleichungen

genügen.

$$
l_{1} \geqq L_{1}, \quad l_{2} \leqq L_{2}
$$

Es genügt daher zu zeigen, dass

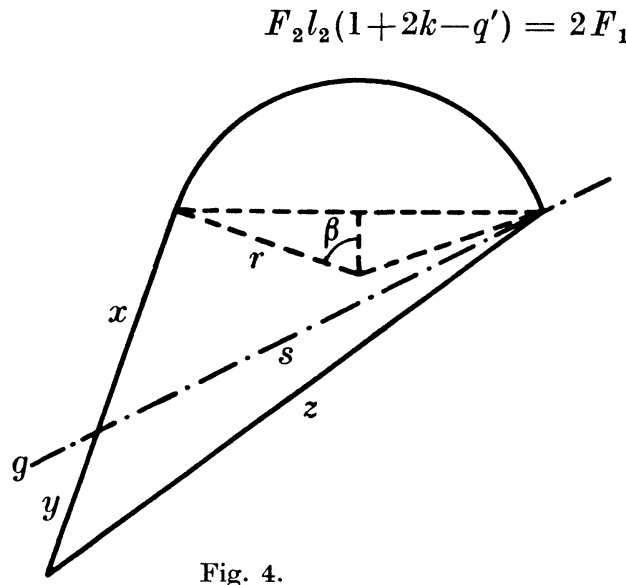

Fig. 4.

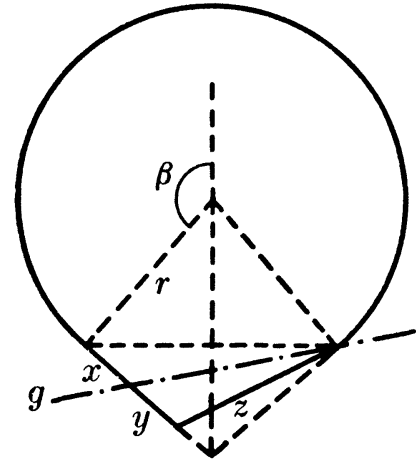

Fig. 5.

positiv ist, wo zur Abkürzung $F_{1} / F_{2}=k$ und $l_{1} / l_{2}=q^{\prime}$ gesetzt ist. Mit den Bezeichnungen der Figur 4 haben wir

also

$$
\begin{array}{ll}
F_{1}=y r \sin ^{2} \beta, & F_{2}=r^{2} \beta-r^{2} \sin \beta \cos \beta+r x \sin ^{2} \beta, \\
l_{1}=y+z, & l_{2}=2 r \beta+x,
\end{array}
$$

$$
\begin{aligned}
F_{2} l_{2}\left(1+2 k-q^{\prime}\right) & =2 F_{1}(2 r \beta+x)+F_{2}(2 r \beta+x-y-z) \\
& =y r^{2}(\sin 2 \beta-2 \beta \cos 2 \beta)+F_{2}(2 r \beta+x+y-z) .
\end{aligned}
$$

Hier ist das zweite Glied des letzten Ausdrucks offensichtlich positiv (vgl. Fig. 4). Die Behauptung ist also jedenfalls richtig, wenn

$$
\sin 2 \beta-2 \beta \cos 2 \beta \geqq 0
$$

gilt, und dies ist der Fall für $0 \leqq \beta \leqq \beta_{1}$, wo $\beta_{1}$ die zwischen $\frac{2}{3} \pi$ und $\frac{3}{4} \pi$ gelegene Nullstelle der linken Seite ist. Ist hingegen $\beta_{1}<\beta \leqq \pi$, also insbesondere $\frac{2}{3} \pi \leqq \beta \leqq \pi$, so hat $\operatorname{man} l_{2} \geqq 2 r \beta \geqq \frac{4}{3} \pi r$ und, wie aus Figur 5 ersichtlich,

$$
l_{1}=y+z \leqq-2 r \operatorname{tg} \beta \leqq 2 r 3^{\frac{1}{2}}<\frac{9}{1^{0}} l_{2},
$$

also $q \leqq q^{\prime}<\frac{9}{10}$. Folglich gilt in diesem Fall sogar

$$
1+2 k-q>\frac{1}{10} \text {. }
$$


Dass 0 tatsächlich die untere Grenze von $1+2 k-q$ ist, folgt aus der Tatsache, dass es Dreiecke gibt, für die sich $q$ bei gegebenem $k$ beliebig wenig von $1+2 k$ unterscheidet. Auf der Seite $A D$ eines Dreiecks $A D C$ sei der Punkt $B$ so gewählt, dass $A B \mid B D=k$ ist. Dann teilt die Gerade $B C$ den Flächeninhalt im Verhältnis $k$, und $q=(A B+A C) /(B D+D C)$ konvergiert offensichtlich gegen $1+2 k$, wenn $C$ bei festen $A$ und $D$ gegen $D$ konvergiert.

4. Es soll nun noch gezeigt werden, dass es eine positive Konstante $\alpha$ derart gibt, dass sogar

$$
1+2 k-q>\alpha F^{2} L^{-4}
$$

für jeden konvexen Bereich mit dem Inhalt $F$ und dem Umfang $L$ gilt.

Wenn $q<\frac{9}{10}$ ist, also, wie gezeigt, insbesondere für $\frac{2}{3} \pi \leqq \beta \leqq \pi$, ist dies klar; denn hier gilt (3), und wegen der isoperimetrischen Ungleichung $4 \pi F \leqq L^{2}$ haben wir

$$
1+2 k-q>\frac{1}{10}(4 \pi F)^{2} L^{-4}>14 F^{2} L^{-4} .
$$

Im folgenden wird daher $0<\beta \leqq \frac{2}{3} \pi$ und $q \geqq \frac{9}{10}$ vorausgesetzt. Dann gilt, wie man mit Hilfe der Taylorschen Formel leicht feststellt,

$$
0<\beta-\sin \beta \cos \beta \leqq 2 \beta^{3},
$$

und wegen $\sin ^{2} \beta<\beta^{2}$ hat man daher

$$
F_{2} \leqq r\left(2 r \beta^{3}+x \beta^{2}\right)=r \beta^{2} l_{2} .
$$

Ferner folgt aus $q \geqq \frac{9}{1^{0}}$

$$
L=(q+1) L_{2} \geqq \frac{19}{10} L_{2} \geqq \frac{19}{10} l_{2} \text {. }
$$

Es werde zunächst $k>1$ angenommen. Aus (1) folgt mit Benutzung von $k F_{2}=F_{1}=y r \sin ^{2} \beta$ und (4)

$$
\begin{aligned}
1+2 k-q & \geqq 1+2 k-q^{\prime} \geqq F_{2}{ }^{-1} l_{2}{ }^{-1} y r^{2}(\sin 2 \beta-2 \beta \cos 2 \beta) \\
& =k F_{2} l_{2}{ }^{-2} r l_{2} F_{2}{ }^{-1} \sin ^{-2} \beta(\sin 2 \beta-2 \beta \cos 2 \beta) \geqq k F_{2} l_{2}{ }^{-2} \varphi(\beta),
\end{aligned}
$$

wo zur Abkürzung

$$
\beta^{-2} \sin ^{-2} \beta(\sin 2 \beta-2 \beta \cos 2 \beta)=\varphi(\beta)
$$

gesetzt ist. Aus (5) und $F=(k+1) F_{2}$ folgt also wegen $k>1$

$$
1+2 k-q>\frac{9}{4} k(k+1)^{-1} F L^{-2} \varphi(\beta)>F L^{-2} \varphi(\beta) \geqq 4 \pi F^{2} L^{-4} \varphi(\beta) .
$$

Es bleibt also zu zeigen, dass $\varphi(\beta)$ in dem betrachteten Intervall $0<\beta \leqq \frac{2}{3} \pi$ eine positive untere Grenze hat. Nun erhält man leicht 
mit Hilfe der Taylorschen Formel die für $0<\beta<\frac{1}{4} \pi$ gültigen Abschätzungen

also

$$
\sin 2 \beta>2 \beta-\frac{4}{3} \beta^{3}, \quad \cos 2 \beta<1-2 \beta^{2}+\frac{2}{3} \beta^{4},
$$

$$
\varphi(\beta)>\beta \sin ^{-2} \beta\left(\frac{8}{3}-\frac{4}{3} \beta^{2}\right)>\frac{4}{3} \quad \text { für } \quad 0<\beta<\frac{1}{4} \pi .
$$

Im Intervall $\frac{1}{4} \pi \leqq \beta \leqq \frac{2}{3} \pi$ hat die Funktion $\sin 2 \beta-2 \beta \cos 2 \beta$ ein Maximum bei $\beta=\frac{1}{2} \pi$ und sonst keine Extrema. Sie nimmt daher ihren kleinsten Wert in einem Endpunkt, und zwar für $\beta=\frac{1}{4} \pi$ an, so dass dieser kleinste Wert gleich 1 ist. Folglich haben wir

$$
\varphi(\beta) \geqq \beta^{-2} \sin ^{-2} \beta>{ }_{4}^{9} \pi^{-2} \quad \text { für } \quad \frac{1}{4} \pi \leqq \beta \leqq \frac{2}{3} \pi,
$$

und daher im ganzen Intervall $0<\beta \leqq \frac{2}{3} \pi$. Wir erhalten somit in diesem Fall

$$
1+2 k-q>9 \pi^{-1} F^{2} L^{-4}>2 F^{2} L^{-4} .
$$

Nun sei $k \leqq 1$. Aus (1), (2) und $z-y<s<l_{2}$ (vgl. Fig. 4) folgt

$$
1+2 k-q>l_{2}{ }^{-1}\left(l_{2}-s\right)=l_{2}^{-1}\left(l_{2}+s\right)^{-1}\left(l_{2}^{2}-s^{2}\right)>\frac{1}{2} l_{2}{ }^{-2}\left(l_{2}{ }^{2}-s^{2}\right) .
$$

Aus der Figur 4 entnimmt man ferner

$$
s^{2}=4 r^{2} \sin ^{2} \beta+4 r x \cos \beta \sin \beta+x^{2},
$$

also wegen $l_{2}=2 r \beta+x$

$$
l_{2}{ }^{2}-s^{2}=4 r^{2}\left(\beta^{2}-\sin ^{2} \beta\right)+4 r x(\beta-\sin \beta \cos \beta) \geqq 4 r^{2}\left(\beta^{2}-\sin ^{2} \beta\right) .
$$

Mit Berücksichtigung von (4) finden wir daher

$$
1+2 k-q>2 r^{2} l_{2}^{-2}\left(\beta^{2}-\sin ^{2} \beta\right)>2{F_{2}}_{2}{ }^{2} 2_{2}^{-4} \beta^{-4}\left(\beta^{2}-\sin ^{2} \beta\right) \text {. }
$$

Nun ist $\beta+\sin \beta>\frac{7}{5} \beta$ und $\beta-\sin \beta>\frac{1}{8} \beta^{3}$ für $0<\beta \leqq \frac{2}{3} \pi$, so dass sich in diesem Fall wegen $F=(k+1) F_{2} \leqq 2 F_{2}$ und (5)

ergibt.

$$
1+2 k-q>2 \cdot \frac{1}{4} \cdot\left(\frac{19}{1} \frac{9}{0}\right)^{4} \cdot \frac{7}{5} \cdot \frac{1}{8} \cdot F^{2} L^{-4}>F^{2} L^{-4}
$$

Die behauptete Ungleichung gilt also in allen Fällen mit $\alpha=1$.

\section{LITERATUR}

1. W. Blaschke, Kreis und Kugel, Leipzig, 1916.

2. J. Favard, Problèmes d'extremums relatifs aux courbes convexes (Premier mémoire), Ann.

Sci. École Norm. Sup. 46 (1929), 345-369. 